\title{
Vertikale Integration und Unternehmenserfolg: eine empirische Untersuchung in der Formel $1^{\dagger}$
}

\author{
Dr. Andreas Hack \\ Lehrstuhl für Innovations- und Gründungsmanagement \\ Technische Universität Dortmund, Otto-Hahn-Straße 4, 4227 Dortmund \\ e-Mail: andreas.hack@uni-dortmund.de \\ Dr. Jörg Schiller \\ Institut für Risikoforschung und Versicherungswirtschaft \\ Ludwig-Maximilians-Universität München, Schackstr. 4/III, 80539 München \\ e-Mail: schiller@bwl.lmu.de
}

Keywords: Vertikale Integration, Unternehmenserfolg, Unterinvestitionsprobleme, Motorsport

JEL: D02, D23, L22

\footnotetext{
${ }^{\dagger}$ Für hilfreiche Anmerkungen danken wir insbesondere zwei anonymen Gutachtern, Ralf Elsas, Oliver Gürtler, Johannes Jaspersen, Peter-J. Jost, Anno Hecker, Barbara Klimaszewski-Blettner, Tobias Kretschmer, Ulrich Lichtenthaler, Werner Neus, Roland Paarz, Paul Raschky, Andre Thimm sowie den Teilnehmern des Offenen Forschungsseminars Management \& Microeconomics an der LMU München, des betriebswirtschaftlichen Forschungskolloquiums der Technischen Universität Dortmund und des Forschungskolloquiums der WHU in Vallendar.
} 


\section{Vertikale Integration und Unternehmenserfolg: eine empirische Untersuchung in der Formel 1}

\section{Überblick}

- Die neue Theorie der Unternehmung befasst sich unter anderem mit der zentralen Frage, wie sich vertikale Integrationsentscheidungen auf das Investitionsverhalten von Unternehmen und deren Gewinne auswirken. Aufgrund vielfältiger Messprobleme, wie beispielsweise unkontrollierbare Umwelteinflüsse, Endogenitätsprobleme oder Schwierigkeiten der Erfolgsmessung, konnte diese Frage empirisch bisher nur unzureichend beantwortet werden.

- Die weitgehend konstanten Wettbewerbsbedingungen und das eindeutige und leicht beobachtbare Erfolgsmaß des Rennerfolgs machen die Formel 1 zu einem idealen Untersuchungsumfeld zur Beantwortung der Frage nach den Erfolgswirkungen vertikaler Integration. In der Formel 1 kann seit dem Jahr 2002 eine Tendenz zur Gründung oder Übernahme von Rennställen durch große Automobilkonzerne beobachtet werden, die bisher nur als Motorenlieferanten auftraten.

- Die vorliegende Untersuchung zeigt, dass die vertikale Vorwärtsintegration von Automobilkonzernen den Teamerfolg in der Formel 1 steigert. Es wird insbesondere deutlich, dass vertikal integrierte Teams höhere Investitionen tätigen und dadurch die Qualität der Fahrer und die technische Qualität der Rennwagen verbessern. Diese Faktoren wirken signifikant auf den Rennerfolg. 


\section{A Einleitung und Forschungsfragen}

Die Erstellung von Gütern oder Dienstleistungen durch Unternehmen wird in der Regel arbeitsteilig organisiert. Entscheidungen hinsichtlich der Leistungstiefe beziehungsweise des Grades der vertikalen Integration der Produktion und des Absatzes sind für Unternehmen vor allem dann von herausragender Bedeutung, wenn Märkte unvollkommen und verschiedene Organisationsformen mit unterschiedlichen Transaktionskosten verbunden sind.

Die theoretische Literatur zur neuen Theorie der Unternehmung, die vor allem durch Coase (1937) initiiert wurde, beinhaltet mittlerweile eine Reihe von sehr unterschiedlichen Ansätzen zur Klärung der beiden zentralen Fragestellungen in diesem Bereich:

1. Welche Transaktionstypen sollten innerhalb eines Unternehmens durchgeführt werden?

2. Welche Auswirkungen hat die vertikale Integration von Transaktionen auf Marktergebnisse wie Preise, Investitionen oder Gewinne?

Besonders hervorzuheben sind dabei transaktionskostentheoretische Überlegungen (Williamson, 1975, 1979, 1985; Klein et al., 1978), Ergebnisse der Property-Rights Theorie (Grossman und Hart, 1986; Hart und Moore, 1990), Ableitungen aus der Prinzipal-Agenten und Team Theorie (Alchian und Demsetz, 1972; Holmström, 1982; Eisenhardt, 1989), sowie Argumente des ressourcenbasierten Strategieansatzes (Penrose, 1959; Wernerfelt, 1984; Barney, 1991).

Empirische Arbeiten belegen weitgehend die theoretischen Überlegungen zur ersten Frage nach dem optimalen Integrationsgrad der Wertschöpfung. Insbesondere die Vorhersage des Transaktionskostenansatzes, dass die Spezifität von Investitionen eine entscheidende Rolle für die Integrationsentscheidung spielt und höhere Spezifität mit einer höheren Wahrscheinlichkeit vertikaler Integration einhergeht, findet umfangreiche Bestätigung. ${ }^{1}$ Ebenso konnte gezeigt werden, dass das in der Prinzipal-Agenten Theorie diskutierte Problem der Messung individueller Leistungsbeiträge von Transaktionspartner bei asymmetrischer Informationsverteilung die Integrationswahrscheinlichkeit erhöht (Mahoney, 1992). Der zweiten zentralen Frage, welche Auswirkungen die vertikale Integration von Transaktionen auf Marktergebnisse wie Preise, Investitionen oder Gewinne hat, wurde in der empirischen Forschung dagegen weit weniger Aufmerksamkeit zuteil (Leiblein et al., 2002). Insbesondere über die unmittelbare Erfolgswirkung der vertikalen Integration auf Unternehmensebene lassen die wenigen empirischen Befunde nur bedingt Rückschlüsse zu (Spiller, 1985; 
Harrigan, 1986; Kerkvliet, 1991; D’Aveni und Ravenscraft, 1994; Poppo und Zenger, 1998; Leiblein et al., 2002). Dies ist darauf zurückzuführen, dass empirische Felduntersuchungen mit nicht zu vernachlässigenden Kontrollproblemen verbunden sind. Zusätzlich ergibt sich in der Regel ein Endogenitätsproblem, das heißt zusätzlich zur Veränderung des Integrationsgrads werden auf Unternehmensebene vielfach andere wichtige strukturelle Entscheidungen getroffen, die eine Analyse der isolierten Wirkung hinsichtlich des Integrationsgrads erschweren. Hinzu kommt das generelle Problem der Erfolgsmessung unterschiedlicher Austauschmechanismen, da objektive Daten hierzu nur schwer zu erhalten sind. So stützen sich die meisten Studien entweder auf reine Kosteneinschätzungen, die den Erfolg einer Unternehmung nicht direkt widerspiegeln, oder nutzen subjektive Beurteilungskriterien wie die Zufriedenheit mit der Qualitätsentwicklung der Produkte (D’Aveni und Ravenscraft, 1994; Poppo und Zenger, 1998).

Zentraler Forschungsschwerpunkt dieser Arbeit ist die Auswirkung der vertikalen Integration auf Unternehmensebene. Die vorliegende Untersuchung beschäftigt sich somit primär mit der Frage, ob die vertikale Integration zentraler Wertschöpfungsaktivitäten unter bestimmten Marktgegebenheiten zu einer Erhöhung der Investitionsneigung und zu einer Verbesserung des Unternehmenserfolgs führt. Um die unmittelbare Wirkung des Integrationsgrads auf Unternehmensebene analysieren zu können, wird die Erfolgswirkung der vertikalen im spezifischen Umfeld des Formel 1-Rennsports bearbeitet. Dort kann seit dem Jahr 2002 eine Tendenz zur Gründung oder Übernahme von Rennställen durch große Automobilkonzerne beobachtet werden, die zuvor als reine Motorenlieferanten für die jeweiligen Teams agierten.

Die zunehmende Integrationstendenz in der Formel 1 kann insbesondere unter Berücksichtigung des Transaktionskostenansatzes und der Prinzipal-Agenten-Theorie erklärt werden. Die Integrationsentscheidung selbst ist jedoch nicht primärer Gegenstand der weiteren Analyse, da die vorhandene Datenbasis keine umfassenderen Analysen zulässt und somit konkrete unternehmensspezifische Einflussfaktoren, wie beispielsweise Kosten- oder Zielfunktionen, die Verhandlungsmacht der beteiligten Parteien sowie die relative Bedeutung der spezifischen Investitionsbeiträge, nur unzureichend erfasst werden können.

Zentrale Vorteile der Analyse des Formel 1-Rennsports ergeben sich vor allem bei der Messung und Interpretation der Daten und empirischen Ergebnisse im Hinblick auf die Auswirkungen der vertikalen Integration. Zunächst existiert mit den erzielten WM-Punkten 
beziehungsweise der WM-Platzierung ein für alle Wettbewerbsteilnehmer objektives, eindeutiges und beobachtbares Erfolgskriterium. Das Wettbewerbsumfeld ist darüber hinaus durch explizite Regeln eindeutig beschrieben und im Betrachtungszeitraum nahezu konstant. Im Weiteren spielen in der Formel 1 strategische Erwägungen, wie beispielsweise die Verhinderung des Marktzutrittes von potentiellen Konkurrenten oder die Erlangung von Marktmacht, eine untergeordnete Rolle, so dass nur eine begrenzte Zahl von wichtigen Wettbewerbs- beziehungsweise Erfolgsfaktoren durch die Integrationsentscheidung beeinflusst werden.

Neben den genannten Vorteilen ist die Analyse der Fragestellung innerhalb der Formel 1 mit zwei wesentlichen Nachteilen verbunden. Zum einen können im Rahmen der vorliegenden Untersuchung nur Daten aus öffentlichen Datenbanken genutzt werden, weil die Formel 1-Teams hinsichtlich der Entwicklung der Rennwagen, der genauen Budgetierung, der Teamprozesse und anderer möglicher Erfolgsdeterminanten strenger Geheimhaltung unterliegen. Da die wichtigsten Erfolgsdeterminanten, wie die Fahrerqualität, die Leistungsfähigkeit des Rennwagens und dessen Zuverlässigkeit jedoch recht gut über die vorliegenden Daten approximiert werden können, dürfte diese Einschränkung keinen nennenswerten negativen Einfluss auf die Zuverlässigkeit der Ergebnisse ausüben. Ein zweiter Nachteil ist dagegen bedeutsamer: Die Fahrerqualität und Leistungsfähigkeit des Rennwagens sind nur schwer voneinander zu trennen. Beide Erfolgsfaktoren beeinflussen sich gegenseitig, so dass die isolierte Erfolgswirkung jeweils nur schwer abzuschätzen ist. Dieses Problem wurde durch die Verwendung alternativer Kennzahlen berücksichtigt. Es zeigte sich, dass die generellen Erkenntnisse für unterschiedliche Berechnungen gelten. An den entsprechenden Stellen werden daher immer nur die Ergebnisse für eine Berechnungsmethode ausführlich angegeben. Auf alternative Formen wird jeweils kurz hingewiesen.

Im Rahmen der vorliegenden Arbeit kann für den Formel 1-Rennsport gezeigt werden, dass die vertikale Vorwärtsintegration von Automobilkonzernen den Teamerfolg steigert. Die genauere Analyse der Wirkungszusammenhänge verdeutlicht vor allem, dass vertikal integrierte Teams deutlich höhere Budgets aufweisen und darüber die Qualität ihrer Fahrer aber auch die technische Qualität ihrer Rennwagen deutlich steigern können. Diese Faktoren wirken ihrerseits wiederum signifikant auf die Erfolgswahrscheinlichkeit bei Grand PrixRennen der Weltmeisterschaft und somit auf den Teamerfolg. 
Die Arbeit gliedert sich im Weiteren wie folgt: In Abschnitt 2 wird zunächst der Wettbewerb im Formel 1-Rennsport grundlegend skizziert und allgemeine Tendenzen zur verstärkten vertikalen Integration beschrieben. Durch die Verbindung des Transaktionskostenansatzes mit der Prinzipal-Agenten-Theorie werden grundsätzliche Vorteile der vertikalen Integration aus theoretischer Sicht analysiert. Aus diesen Überlegungen wird dann im zweiten Schritt ein Pfadmodell zur Wirkungsweise des Integrationsgrads auf wichtige Erfolgsfaktoren innerhalb der Formel 1 abgeleitet, um die spezifische Wirkung der Integrationsentscheidung auf Unternehmensebene beurteilen zu können. In Abschnitt 3 erfolgt eine eingehende empirische Analyse des zuvor hergeleiteten Pfadmodells. Die allgemeinen Auswirkungen des Integrationsgrads auf den Unternehmenserfolg werden abschließend in $A b$ schnitt 4 diskutiert und zusammengefasst.

\section{B Theoretische Grundlagen und Hypothesen}

\section{Grundlagen des Formel 1-Rennsports}

Die Formel 1 ist eine seit 1950 vom Automobilweltverband FIA (Fédération Internationale de l'Automobile) jährlich veranstaltete Rennserie. In jedem der 18 Einzelrennen kämpfen 11 Rennställe (Teams) mit jeweils 2 typgleichen Fahrzeugen um eine festgelegte Anzahl an Weltmeisterschaftspunkten (WM-Punkten). ${ }^{2}$ Derjenige Fahrer, der in der Summe aller gewerteten Rennen am Saisonende die höchste Punktzahl erreicht, gewinnt die Fahrerweltmeisterschaft. Als weiteres Wertungskriterium wird dasjenige Team als Konstrukteursweltmeister ausgezeichnet, dessen Fahrer über eine Saison die meisten WM-Punkte erzielt haben.

Wichtigstes Ziel der Rennställe und ihrer Eigentümer ist die erfolgreiche Teilnahme ihrer Autos im Gesamtklassement und damit der Gewinn von WM-Punkten. Auch aus Sicht der wichtigsten Zulieferer und Sponsoren der Rennställe, wie beispielsweise der Motorenlieferanten BMW oder Daimler, steht aus Gründen des Aufbaus und der Verbesserung des Markenimages die erfolgreiche Teilnahme der von ihnen unterstützen Rennställe im Vordergrund. ${ }^{3}$ Damit ergibt sich ein objektives und beobachtbares Erfolgskriterium, an dem die Wirkung einer vertikalen Integrationsentscheidung eindeutig gemessen werden kann. 
Die Teilnahme in der Formel 1 ist nur bestehenden Teams gestattet oder neuen Bewerbern, wenn diese eine Garantiesumme von 48 Mio. US-Dollar hinterlegen und alle sportlichen wie technischen Auflagen erfüllen. Dies bedeutet einen beschränkten Zugang zur Formel 1 und damit ein kontrolliertes Wettbewerbsumfeld in Bezug auf die Konkurrenzsituation. Damit kommen strategischen Überlegungen zur Beschränkung der Wettbewerbsintensität oder dem Aufbau von Marktmacht durch einzelne Teams kaum Bedeutung zu.

Die gesamte Formel 1 unterliegt strengen sportlichen und technischen Wettbewerbsregeln. ${ }^{4}$ Sie dienen dem Zweck, möglichst einheitliche Wettbewerbsbedingungen für alle Teilnehmer zu schaffen. Neben Regeln zum Fahrer gelten insbesondere zur technischen Ausprägung der Rennwagen detaillierte Regelungen. Jeder Rennstall entwickelt für jede Saison einen spezialisierten Rennwagen. Während die Entwicklung des Fahrzeugchassis bisher eine zentrale Kernkompetenz der Rennställe darstellt, werden andere technische Komponenten, wie der Motor, die Reifen, der Kraftstoff, das Getriebe oder die Fahrzeugelektronik in der Regel von den Rennställen im Rahmen von so genannten „technischen Partnerschaften“ fremdbezogen. ${ }^{5}$

Das Reglement der Formel 1 verfolgt vor allem das Ziel der Geschwindigkeits- beziehungsweise Leistungsreduzierung. Formel 1-Motoren waren zunächst auf eine hohe Leistungsbereitstellung ausgelegt. Um die Geschwindigkeit der Rennwagen zu reduzieren, wurde seit 1986 sukzessive der zulässige Motorenhubraum herabgesetzt, so dass seit der Saison 2006 nur noch Motoren mit einem Hubraum von maximal $2.400 \mathrm{~cm}^{3}$ eingesetzt werden dürfen. Bedingt durch die andauernde, intensive Entwicklung sind weitere Leistungssteigerungen im Motorenbereich sehr kostenintensiv. Stärkere Bedeutung kommt mittlerweile der Motorenhaltbarkeit zu. Mit Beginn der Saison 2004 durfte ein Rennmotor während eines Grand Prix-Wochenendes nicht ausgetauscht werden. Seit dem Jahr 2005 müssen die Motoren jeweils zwei Rennwochenenden halten.

Bedingt durch die starke Spezialisierung der Arbeitsprozesse und die kurzen Produktzyklen ergibt sich im Rahmen der Erst- und Weiterentwicklung des Rennwagens ein starker Koordinationsbedarf zwischen den unterschiedlichen Einzelaktivitäten. Konflikte treten insbesondere bei der Dimensionierung des Motors auf. So benötigt beispielsweise ein leistungsstärkerer Motor eine größer dimensionierte Kühlung, die wiederum eine schlechtere Aerodynamik des Fahrzeugchassis impliziert. Der Organisationsstruktur und dem Teammana- 
gement kommt bei der Lösung auftretender Koordinierungsprobleme eine besondere Bedeutung zu (Jenkins, 2004).

Bis zum Jahr 2002 bezogen Formel 1-Rennställe ihre Motoren fast ausschließlich von großen Automobilkonzernen. Eine Ausnahme bildete Ferrari, die ihre Motoren stets selbst erstellten. Infolge der Dominanz des stark vertikal integrierten Ferrari-Teams in den Jahren 2000-2004 gingen Automobilkonzerne wie Ford (im Jahr 2000) oder Toyota (im Jahr 2002) dazu über, eigene Rennställe zu gründen, während beispielsweise Renault (im Jahr 2002), BMW (im Jahr 2005) oder Honda (im Jahr 2006) etablierte Rennställe im Sinne einer Vorwärtsintegration übernahmen.

Zusammenfassend stellt die Formel 1 einen Markt dar, der als Analyseobjekt zur empirischen Beantwortung der Frage nach den Auswirkungen der vertikalen Integration sehr gut geeignet ist. Erstens handelt es sich um einen stark reglementierten Markt mit wenigen unkontrollierbaren Umwelteinflüssen. Zweitens wirken nur wenige zentrale Faktoren auf den Erfolg der Rennställe, wie Fahrer und Rennwagen. Drittens kann ein eindeutiges Erfolgskriterium beobachtet werden. Viertens haben strategische Erwägungen, wie beispielsweise die Erlangung von Marktmacht, keine oder eher eine untergeordnete Bedeutung. Und fünftens handelt es sich um einen Wettbewerb, in dem integrierte als auch nicht-integrierte Teams unmittelbar gegeneinander antreten, so dass Erfolgsunterschiede direkt beobachtet werden können. Darüber hinaus ist die Wirkung des vertikalen Integrationsgrads auf wichtige Marktergebnisse beobachtbar.

II Einfluss des Integrationsgrads auf den Erfolg in der Fahrerweltmeisterschaft

Da der Integrationsgrad keinen unmittelbaren Einfluss auf das Renngeschehen in einem Grand Prix hat, wird im Folgenden ein Kausalmodell hergeleitet, welches den Einfluss der Integration auf das Renngeschehen über Mediatorvariablen darstellt. Dabei werden ausgehend von dem Erfolgsmaß der WM-Punktzahl und der WM-Platzierung die einzelnen Kausalzusammenhänge sukzessive über Hypothesen erarbeitet. Auf einer ersten Stufe wird der unmittelbare Einfluss der Fahrer- und Rennwagenqualität auf den WM-Erfolg diskutiert. Diese Erfolgsdeterminanten werden wiederum durch das dem Team zur Verfügung stehende Budget sowie die Stärke der Kooperationsbeziehung zwischen Motorenhersteller und Rennstall beeinflusst. Diese Zusammenhänge werden auf einer zweiten Stufe dargestellt. Abschließend wird auf einer dritten Stufe der Einfluss des Integrationsgrads auf das Budget 
und die Kooperationsbeziehung theoretisch mit Hilfe des Transaktionskostenansatzes und der Prinzipal-Agenten Theorie begründet. Abschließend werden die einzelnen Kausalzusammenhänge in einem umfassenden Pfadmodell integriert.

\section{Fahrer und Rennwagen als kritische Erfolgsdeterminanten in der Formel 1}

Die Bedeutung des Piloten für den Rennerfolg liegt nicht nur in seinen eigentlichen Fahrkünsten, sondern auch in der Art und Weise, wie er im gesamten Team die Entwicklung des Rennwagens vorantreibt und die Motivation des Teams hochhält. Die Qualität eines Rennfahrers wirkt sich über seine Fahrqualität und Rennintelligenz direkt auf den Rennerfolg aus. Dagegen wirken Kommunikationsfähigkeit und technisches Verständnis, welche zur Entwicklung eines überlegenen Rennwagens beitragen, eher indirekt auf die Fahrerwertung.

Hypothese 1: Je besser die Fahrerqualität des Formel 1-Piloten, desto mehr WM-Punkte werden eingefahren.

Neben dem Piloten spielt der Rennwagen die entscheidende Rolle im eigentlichen Renngeschehen. Dessen Qualität setzt sich aus seiner Leistungsfähigkeit und Zuverlässigkeit während der Rennsaison zusammen. Die absolute Leistungsstärke eines Rennwagens bestimmt maßgeblich die möglichen schnellsten Rundenzeiten in einem Grand Prix und spielt sowohl beim Start als auch bei freier Rennstrecke eine große Rolle. Zudem übt die Beschleunigungsfähigkeit des Rennwagens einen wesentlichen Einfluss auf die Möglichkeit für Überholmanöver in einzelnen Rennen aus.

Hypothese 2: Je höher die Leistungskraft des Rennwagens, desto mehr WM-Punkte werden eingefahren.

Die Zuverlässigkeit des Rennwagens ist ebenfalls von zentraler Bedeutung. Der Ausfall eines Rennwagens in einem einzigen Grand Prix kann für die Platzierung in der Gesamtfahrerwertung ausschlaggebend sein. Insbesondere die technische Zuverlässigkeit des Motors ist durch das aktuelle Reglement, nach dem ein Motor nur noch alle zwei Rennwochenenden ausgewechselt werden darf, in den Mittelpunkt des Interesses gerückt. ${ }^{6}$

Hypothese 3: Je höher die Zuverlässigkeit des Rennwagens, desto mehr WM-Punkte werden eingefahren. 
2. Budget und Kooperationsdauer und ihr Einfluss auf die kritischen Erfolgsfaktoren in der Formel 1

Der Wettbewerb der Rennställe um die Verpflichtung leistungsfähiger Formel 1-Piloten ist sehr intensiv. Die Piloten entsprechen nach der ressourcenorientierten Sichtweise wettbewerbsrelevanten Kernkompetenzen (Grant, 1991). Dass es sich um eine wertvolle Ressource handelt, wurde bereits unter Hypothese 1 dargestellt. Die Knappheit der Ressource liegt an den speziellen Zulassungsbedingungen für Piloten zur Formel 1. Laut FIA Reglement dürfen nur solche Piloten an der Formel 1 teilnehmen, die eine sogenannte Super Licence halten. ${ }^{7}$ Daraus folgt ein Wettbewerb um eine sehr knappe und wertvolle Ressource. Da die Aufgaben der Piloten sowie der Arbeitsaufwand im Wesentlichen in allen Rennställe gleich bleibt, können sich die Rennställe die besten Piloten nur dann dauerhaft aneignen, wenn sie wettbewerbsfähige Gehälter zahlen.

Hypothese 4a: Je höher das Budget eines Rennstalls ist, desto besser ist die Qualität des Formel 1-Piloten.

Ähnliches gilt für die Entwicklung der Rennwagen. Kritische Ressourcen sind hier die Design- und Entwicklungsmitarbeiter, die vor der Herausforderung stehen, kontinuierlich auch während des Rennbetriebs - inkrementelle Verbesserungen des Rennwagens vorzunehmen. Zudem müssen vorgegebene Renntermine eingehalten werden, so dass die Designund Entwicklungsteams stets unter einem hohen Zeitdruck arbeiten. Die optimalen Teammitarbeiter sind Ingenieure, die nicht nur einzelne Schritte der Entwicklung des Rennwagens beherrschen, sondern den gesamten Entwicklungszyklus überblicken können. Damit handelt es sich ebenfalls um wertvolle und knappe Ressourcen, die zudem häufig zwischen den einzelnen Rennställen wechseln und sehr mobil sind (Jenkins et al., 2005). Leistungsanreize können auch hier insbesondere über Gehälter gesetzt werden.

Weitere Qualitätssteigerungen in der Rennwagentechnologie sind nur durch kapitalintensive Investitionen in Forschungs- und Entwicklungseinrichtungen möglich. Große Rennställe wie Honda, Renault oder Toyota wenden alleine zwischen 120 und 180 Mio. € für die Entwicklung und Produktion der Rennmotoren auf (Renner, 2005). Daraus kann gefolgert werden, dass sowohl die Leistungsstärke als auch die Zuverlässigkeit der Rennwagen nur durch dauerhaft hohe Investitionen gesteigert werden kann. 
Hypothese 4b: Je höher das Budget eines Rennstalls ist, desto höher ist die Leistungskraft des Rennwagens.

Hypothese 4c: Je höher das Budget eines Rennstalls ist, desto höher ist die Zuverlässigkeit des Rennwagens.

Aufgrund der in Abschnitt 2.1 beschriebenen Reglementänderungen der FIA und der bereits sehr hohen technischen Reife des Antriebs und der Chassis, ist es derzeit kaum noch möglich, durch die isolierte Entwicklung einzelner Komponenten ein überlegenes Auto zu produzieren. Vielmehr spielt, verstärkt durch die kurzen Produktzyklen, die genau abgestimmte und integrierte Entwicklung von Antrieb und Aerodynamik eine immer bedeutendere Rolle.

Empirische Studien zur Zusammenarbeit zwischen verschiedenen Forschungs- und Entwicklungsteams kommen zu der Erkenntnis, dass aufgrund der Aufgabeninterdependenzen und der laufenden Anpassungserfordernisse während des Entwicklungsprozesses ein enger Informationsaustausch zwischen den Teams einen wesentlichen Erfolgsfaktor darstellt (Ancona und Caldwell, 1992; Hoegl et al., 2004). Darüber hinaus gewinnt ein gemeinsames Verantwortungsgefühl für die Endergebnisse beider Teams an Bedeutung für den Projekterfolg (Klein und Mulvey, 1995; McDonough, 2000; Hoegl et al., 2004). Durch die Dauer der Kooperation zwischen den einzelnen Entwicklungsteams kann der Informationsaustausch durch persönliche Beziehungen als auch durch formalisierte Prozesse verbessert werden. Zudem kann davon ausgegangen werden, dass sich im zeitlichen Ablauf ein „Wir-Gefühl“ zwischen den eigentlich unabhängigen Entwicklungsteams einstellt.

Hypothese 5a: Je länger die Kooperationsdauer zwischen Rennstall und Motorenhersteller ist, desto höher ist die Leistungskraft des Rennwagens.

Hypothese 5b: Je länger die Kooperationsdauer zwischen Rennstall und Motorenhersteller ist, desto höher ist die Zuverlässigkeit des Rennwagens.

3. Der Einfluss des Integrationsgrads auf die Investitionsneigung und Kooperationsdauer der Rennställe

Motive für die Erhöhung des vertikalen Integrationsgrads sind vielfältig und reichen von strategischen Überlegungen, wie dem Aufbau beziehungsweise der Verstärkung von Ein- 
trittsbarrieren für potentielle Konkurrenten, über Preisvorteile bei der Beschaffung beziehungsweise der Realisierung von positiven Skaleneffekten bis hin zur Reduzierung von Marktunsicherheiten (Chatterjee, 1991; Mahoney, 1992). Neben der Property-Rights Theorie (Grossman und Hart, 1986; Hart und Moore, 1990) stellt der Transaktionskostenansatz (Klein et al., 1978; Williamson, 1985) einen der wichtigsten Erklärungsansätze für vertikale Integrationsentscheidungen dar. Gemeinsamer Ausgangspunkt beider Konzepte sind unvollständige Verträge, die aus der hohen Komplexität des Markumfeldes bzw. aus der Nicht-Verifizierbarkeit relevanter Kooperationsfaktoren resultieren (Simon, 1978; Grossman und Hart, 1986), und spezifische Investitionen.

Im Rahmen des Transaktionskostenansatzes müssen sich Unternehmen gegen opportunistisches Verhalten ihrer Transaktionspartner absichern. Rein vertragliche Lösungsansätze führen in diesem Kontext vor allem dann zu Problemen, wenn von Kooperationspartnern transaktionsspezifische Investitionen getätigt werden müssen, die den Kooperationsertrag beeinflussen, jedoch außerhalb der spezifischen Kooperation an Wert einbüßen (Williamson, 1979; Spiller, 1985; Joskow, 1988). Bei hoher Spezifität besteht somit die Gefahr, dass die Vertragspartner im Rahmen von kostspieligen und somit ineffizienten Nachverhandlungen versuchen, sich die entstandene Quasi-Rente anzueignen. Die Gefahr einer so genannten Hold-Up Situation senkt unmittelbar ex-ante Anreize der Transaktionspartner, in spezifische Ressourcen zu investieren. Folge ist ein Unterinvestitionsproblem, welches durch vertikale Integration tendenziell besser gelöst werden kann als durch einen unvollständigen Kooperationsvertrag. ${ }^{8}$

Da Eigentümer über residuale Kontrollrechte hinsichtlich der jeweiligen Vermögensgegenstände verfügen, können diese andere Personen von der Nutzung dieser Vermögensgegenstände ausschließen. Wie im Transaktionskostenansatz wird auch bei der PropertyRights Theorie die Quasi-Rente einer Transaktion durch spezifische Investitionen beeinflusst. Im Rahmen der effizienten Nachverhandlungen wird diese gemäß dem jeweiligen Eigentumsverhältnis an relevanten Vermögensgegenständen aufgeteilt, so dass die Eigentumsstruktur direkt die ex-ante Investitionsanreize der Kooperationspartner determiniert. Folglich sollte gemäß der Property-Rights eine Partei alle relevanten Vermögensgegenstände besitzen, wenn die Investitionstätigkeit dieser Partei von herausragender Bedeutung für die Kooperationsbeziehung bzw. für die Höhe der Quasi-Rente ist. 
Sowohl der Transaktionskostenansatz als auch die Property-Rights Theorie geben einen guten Einblick in die Rolle beziehungsspezifischer Investitionen, sie vernachlässigen aber größtenteils das Problem der Leistungsmessung und -bewertung.

Deren Effekte wurden insbesondere in der Prinzipal-Agenten und der Team Theorie diskutiert (Alchian und Demsetz, 1972; Holmström, 1982; Eisenhardt, 1989). Wenn die individuelle Leistung einzelner Akteure nicht beobachtbar beziehungsweise kontrahierbar ist, werden durch explizite, unvollständige Verträge in der Regel suboptimale Leistungsanreize gesetzt, so dass es zum moralischen Risiko und Trittbrettfahrerproblemen kommen kann. In Hinblick auf das moralische Risiko können im Rahmen der vertikalen Integration vertragliche durch hierarchische Anreize und zusätzliche Kontroll- und Überwachungsaktivitäten ergänzt werden. Insbesondere können sich Weisungen vorgesetzter Instanzen innerhalb einer Organisation auf nicht-kontrahierbare Größen beziehen. Im Weiteren kann dem Trittbrettfahrerproblem in unternehmensinternen Teams durch Gruppendruck und gegenseitige Kontrolle besser begegnet werden. ${ }^{9}$

Ein hoher Integrationsgrad ist somit vor allem dann von Vorteil, wenn sich die relative Bedeutung der Investitionsentscheidung einer Partei sehr hoch ist (Property-Rights Theorie) bzw. wenn die Austauschbeziehung durch eine hohe Spezifität (Transaktionskostenansatz) und hohe Messprobleme (moralisches Risiko und Trittbrettfahrerprobleme) gekennzeichnet ist. Bei Transaktionen in der Formel 1 sind vor allem die letzteren Faktoren der Spezifität und Messprobleme von herausragender Bedeutung. So ist davon auszugehen, dass sich ein speziell auf die Aerodynamik eines McLaren entwickelter Mercedes-Motor nicht ohne Leistungsverluste in ein anderes Chassis einbauen lässt. Zudem kann gerade im Hochtechnologiebereich der Einsatz unternehmensexterner Partner bei ihren Entwicklungsanstrengungen nur unzureichend bestimmt werden. Auch die Leistungskraftsteigerungen oder eine Erhöhung der Zuverlässigkeit des Rennwagens kann aufgrund der eng aufeinander abgestimmten Teilkomponenten nicht einem Entwicklungsteam individuell zugeschrieben werden. Darüber hinaus hat der Formel 1-Erfolg mittlerweile für Motorenhersteller hinsichtlich der Stärkung des Markenimages an Bedeutung gewonnen, während die Ziele der Rennställe im Zeitverlauf unverändert geblieben sind. Die Erklärungskraft der Property-Rights Theorie wird jedoch im konkreten Fall der Formel 1 unter anderem dadurch gemindert, dass die zunehmende Bedeutung des Markenimages nicht unbedingt mit einer höheren relativen Bedeutung der Investition durch Motorenhersteller einhergehen muss. Darüber hinaus ist das 
Markenimage von Automobilherstellern nicht transferierbar, so dass sich durch eine Veränderung der Eigentumsstruktur nur bedingt Investitionsanreize verändern.

Insgesamt ist die Formel 1 ist jedoch ein Markt, in dem ein stark ausgeprägtes Unterinvestitionsproblem bei marktlicher Kooperation bestehen müsste. Da durch die vertikale Integration nur vernachlässigbare Skalenerträge erzielt werden, jedoch bessere Leistungsanreize gesetzt werden können, müsste die Investitionsneigung vertikal integrierter Teams deutlich über denen nicht-integrierte Teams liegen. ${ }^{10}$

Hypothese 6: Vertikal integrierte Teams verfügen über ein höheres Teambudget als nichtintegrierte Teams.

Neben einem positiven Einfluss der vertikalen Integrationsentscheidung auf das Budget eines Rennstalls, ist auch von einem Einfluss auf die Dauer der Kooperationsbeziehung zwischen Rennstall und Motorenlieferant auszugehen. Gerade in Transaktionsbeziehungen mit hoher Spezifität, ist die Beendigung der Kooperation für vertikal integrierte Teams mit höheren Wechselkosten verbunden als für nicht-integrierte Teams, weil Prozesse und Organisationsstruktur der Transaktionspartner spezifisch aufeinander angepasst werden. Auch wenn die Investitionskosten zum Zeitpunkt der Wechselentscheidung versunken sind, verlieren die Kooperationspartner eines vertikal integrierten Teams die Quasi-Rente, die aus den transaktionsspezifischen Investitionen resultiert. Da nicht-integrierte Teams aufgrund des Unterinvestitionsproblems in geringerem Umfang transaktionsspezifische Investitionen tätigen werden und ihre Abläufe und Struktur weniger stark an den Kooperationspartner anpassen, sind deren Wechselkosten verglichen zu integrierten Teams tendenziell geringer. Folglich sinkt bei integrierten Teams die Wahrscheinlichkeit eines Wechsels des Motorenlieferanten.

Hypothese 7: Vertikal integrierte Teams haben eine längere Kooperationsdauer als nichtintegrierte Teams.

Aus den Hypothesen lässt sich nun ein Kausalmodell ableiten, welches den Zusammenhang zwischen Integrationsgrad und WM-Punkten verdeutlicht. Da die inhaltliche und theoretische Ableitung der Hypothesen gezeigt hat, dass der Einfluss des Integrationsgrads auf die WM-Punkte über verschiedene Moderatorvariablen ausgeübt wird, bietet sich im Weiteren kein Totalmodell sondern vielmehr ein Pfadmodell an, welches die einzelnen Moderatorbeziehungen besser verdeutlicht: 


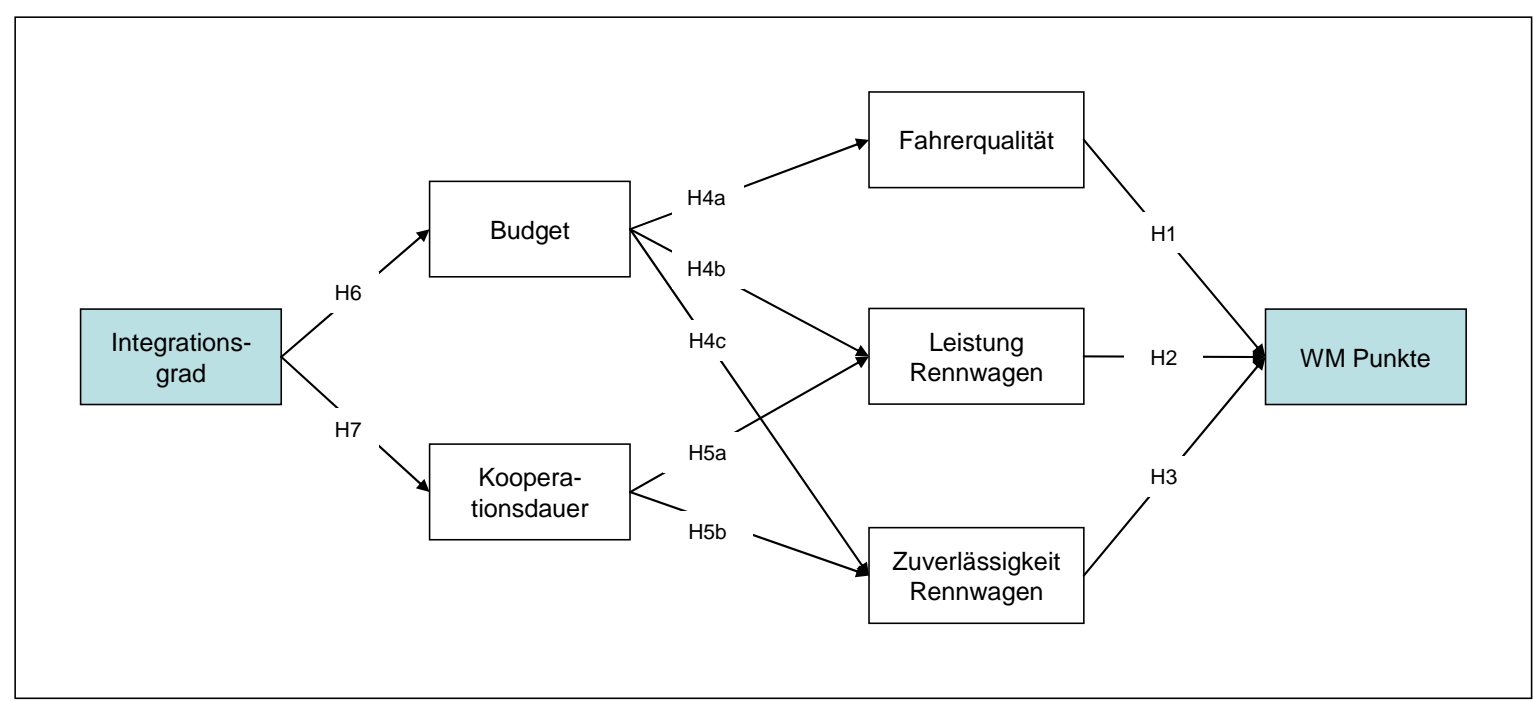

\section{Empirische Analyse}

I Datengrundlage

Grundlage der empirischen Untersuchung sind die Einzelrennergebnisse aus 88 Grand Prix Rennen der Jahre 2002 bis 2006 der Rennserie Formel 1 des Automobilweltverbands FIA. ${ }^{11}$ Die kleinste Untersuchungseinheit ist der einzelne Fahrer eines Teams. Im Verlauf der gesamten Rennsaison dürfen die Teams vier Fahrer einsetzen. ${ }^{12}$ In die Untersuchung wurden pro Team und pro Rennsaison jeweils zwei Fahrer einbezogen und zwar diejenigen Fahrer, die in der jeweiligen Rennsaison für jedes Team in der Einzelfahrerwertung die meisten Punkte gewonnen hatten. Da die Anzahl der Teams, die in jeder Rennsaison zugelassen wurden, zwischen zehn und elf schwankte, konnten insgesamt 104 Einzelfahrerwertungen in die weitere Analyse eingehen. ${ }^{13}$ Alle Daten wurden öffentlich zugänglichen Datenquellen entnommen oder aus diesen abgeleitet. ${ }^{14}$

\section{Operationalisierung der Variablen}

Die im rekursiven Pfadmodell zur Erklärung des Rennerfolgs dargestellten Variablen gilt es im Folgenden zu spezifizieren. Neben der abhängigen Variable Rennerfolg werden die verschiedenen Mediatorvariablen Fahrerqualität, Leistung und Zuverlässigkeit des Rennwagens sowie Budget des Rennstalls und Kooperationsdauer mit dem Motorenlieferant betrachtet. Als unabhängige Variable wird der Grad der vertikalen Integration zwischen 
Rennstall und Motorenlieferant definiert. Neben den erklärenden Variablen, für die im Pfadmodell Hypothesen formuliert wurden, werden mit der Wahl des Reifenherstellers und der Teamdauer zwei Kontrollvariablen berücksichtigt.

\section{Erfolgsvariable/ Abhängige Variable}

Betrachtet man die jeweiligen Zielsetzungen von Rennställen und Motorenlieferanten, so ergeben sich letztendlich die gemeinsamen übergeordneten Ziele des Erfolgs ihrer Fahrer in den einzelnen Grand Prix-Rennen sowie der Gewinn der Gesamtweltmeisterschaft. Hier kann zwischen der Fahrer- und der Konstrukteurswertung unterschieden werden, wobei der Gewinn der Fahrerwertung aufgrund der höheren Imagewirkung für Rennstall und Motorenlieferant eine höhere Bedeutung hat. Als abhängige Variablen wurden für die vorliegende Untersuchung die Punktzahl eines Fahrers (WM-Punkte Fahrer) sowie die Punktzahl eines Teams (WM-Punktzahl Konstrukteur) über alle Grand Prix Rennen einer Saison erhoben. ${ }^{15}$ Damit liegt die abhängige Variable auf metrischem Skalenniveau vor.

\section{Mediatorvariablen}

Die Variablen mit einem vermuteten direkten Erfolgseinfluss auf die WM-Punktzahl wurden wie folgt operationalisiert:

Die Fahrerqualität wurde als addierte Gesamtpunktzahl gemessen, die der Fahrer in den letzten beiden Rennsaisons vor Saisonbeginn eingefahren hat. Nimmt der Fahrer zum ersten Mal in der Klassifikation teil, so werden ihm null Punkte zugerechnet. Das Problem der Autokorrelation spielt hier nur eine untergeordnete Rolle, da die einzelnen Fahrer im Untersuchungszeitraum in unterschiedlichem Ausmaß den Rennstall wechselten. ${ }^{16}$

Als Indikator für die Leistungsfähigkeit des Rennwagens wurde die schnellste Rundenzeit eines jeden Fahrers in jedem Grand Prix gemessen und als prozentuale Abweichung zur insgesamt schnellsten Rundenzeit des jeweiligen Grand Prix berechnet. Diese prozentualen Abweichungen wurden daraufhin über die gesamte Rennsaison für jeden Fahrer addiert. Um den Fahrereinfluss auf die schnellste Rundenzeit zu eliminieren, wurden die prozentualen Abweichungen der Fahrer eines baugleichen Rennwagens addiert und durch die entsprechende Anzahl der Fahrer geteilt. ${ }^{17}$ 
Die technische Ausfallquote pro Rennsaison wurde als Indikator der Zuverlässigkeit des Rennwagens herangezogen. Sie gibt an, in wie viel Prozent der Rennen, an denen ein Fahrer teilgenommen hat, sein Fahrzeug aufgrund technischer Defekte ausgeschieden ist. Ein Ausscheiden durch Unfall oder Disqualifizierung wurde nicht berücksichtigt.

Die Variablen, die einen Einfluss auf die oben genannten Variablen ausüben, wurden wie folgt spezifiziert:

Das Budget eines Teams umfasst alle finanziellen Mittel in Euro, die zur Aufrechterhaltung des Rennbetriebs des jeweiligen Teams in der betrachteten Rennsaison zur Verfügung stehen. Die genaue Erhebung der Teambudgets ist äußerst schwierig und unterliegt hohen Ungenauigkeiten, da die Teams ihre tatsächlichen Budgets zum einen nicht preisgeben, zum anderen auch selbst nur schätzen können. So werden beispielsweise im Falle technischer Partnerschaften die Komponenten von den Partnern unentgeltlich zur Verfügung gestellt. Informationsdienste müssen die potenziellen Kosten der Bereitstellung dieser Komponenten schätzen und unterliegen somit starken Unsicherheiten. Da die Studie auf Budgetschätzungen einer Quelle zurückgreift, dürften die Budgetzahlen, wenn auch nicht absolut, so jedoch in ihrer Relation zueinander korrekt sein. ${ }^{18}$

Unter Kooperationsdauer verstehen wir die Anzahl der Jahre, die ein Formel 1-Team vor Saisonbeginn ohne Unterbrechung bereits mit einem spezifischen Motorenlieferant zusammengearbeitet hat. Dabei spielt es keine Rolle, ob es sich um ein integriertes oder ein nichtintegriertes Team handelt.

\section{Kontrollvariablen}

Als Kontrollvariablen werden in die weitere Analyse der die Teamdauer und der Reifenzulieferer einbezogen. Besondere Bedeutung für den Erfolg innovativer Projekte wird in der der Teamarbeit zugeschrieben (Hoegl und Gemünden, 2001). Gerade in der Formel 1, die durch extrem kurze Produktentwicklungszyklen gekennzeichnet ist, stellt die Zusammenarbeit des Teams einen zentralen Erfolgsfaktor dar (Jenkins et al., 2005). Zudem sind Routinen zur Entwicklung neuer Rennwagen von besonderer Bedeutung. Die Dauer und Beständigkeit eines Teams im Markt stellen somit wichtige Erfolgsfaktoren dar. Wir definieren die Teamdauer über die Anzahl der Jahre, die ein Formel 1-Team vor Saisonbeginn bereits an Grand Prix Rennen ohne Unterbrechung teilgenommen hat. Als weiterer in der Rennpraxis oft diskutierter Erfolgsfaktor wird die Auswahl des Reifenlieferanten gesehen. In der 
Formel 1-Rennserie waren im Betrachtungszeitraum zwei Reifenhersteller aktiv. Daher nimmt die Variable Reifen die Werte 0 für Michelin und 1 für Bridgestone an.

\section{Unabhängige Variable}

Der Begriff der vertikalen Integration wurde in der theoretischen Literatur eingehend diskutiert, wobei sich zwei wesentliche Ansichten gegenüberstehen. Grundsätzlich stellt vertikale Integration das Eigentum und die Kontrolle über alle benachbarten Stufen eines Produktions- oder Distributionsprozesses dar. Williamson (1975) stellt dabei die Produktion durch eigene Mitarbeiter in den Vordergrund, wobei das Eigentum an den Produktionsanlagen nicht gegeben sein muss. Grossman und Hart (1986) und Hart und Moore (1990) verweisen dagegen auf die besondere Bedeutung des Eigentums an den Produktionsanlagen. Zudem werden in der Literatur auch mögliche hybride Formen der vertikalen Integration diskutiert, die von reinen Marktbeziehungen über kurzfristige Verträge, langfristige Verträge, Joint Ventures bis hin zur Hierarchie reichen. Da das Ausmaß des Eigentums und der Kontrolle der einzelnen Produktionsfaktoren zwischen den Kooperationspartnern Rennstall und Motorenhersteller nicht beobachtet werden kann und auch der Grad der Eigenerstellung von Chassis und Motor von den Herstellern nicht offengelegt wird, ist für diese Untersuchung aus Gründen der Praktikabilität die finanzielle Verflechtung der Kooperationspartner ausschlaggebend.

Der vertikale Integrationsgrad des Teams wurde daher als nominal skalierte Variable definiert, die die Werte 0 (nicht integriert) und 1 (integriert) annehmen kann. Ein Team gilt dann als integriert, wenn der Motorenlieferant aufgrund einer Mehrheitsbeteiligung am Rennstall die Strategie und das Tagesgeschäft des Teams weitestgehend autonom bestimmen kann. ${ }^{19}$

\section{Ergebnisse}

Von den 104 betrachteten Einzelfahrerwertungen der letzten fünf Jahre entfallen 61,5 \% (64 Wertungen) auf nicht-integrierte Teams und 38,5\% (40 Wertungen) auf integrierte Teams. Fahrer integrierter Teams konnten über den Betrachtungszeitraum im Schnitt pro Rennsaison 46,4 Punkte gewinnen, im Vergleich zu 20,25 Punkten, die Fahrer nichtintegrierter Teams einfuhren. Diese erste visuelle Betrachtung der Gruppenunterschiede bekräftigt unsere Hypothese, die die Überlegenheit integrierter Teams postuliert. Die statis- 
tische Überprüfung der Mittelwertunterschiede zwischen Fahrern integrierter und nichtintegrierter Teams $(\mathrm{t}=-3,753$; $\mathrm{p}<0,001)$ bestätigt diesen Eindruck. ${ }^{20}$

Um den kausalen Zusammenhang zwischen dem Grad der vertikalen Integration und der WM-Punktzahl zu analysieren, wurde in einem nächsten Schritt das in Abschnitt 2.2 theoretisch erarbeitete Pfadmodell berechnet. ${ }^{21}$ Tabelle 1 zeigt die deskriptiven Statistiken und Korrelationen der einzelnen Pfadvariablen.

Tabelle 1: Deskriptive Statistik und Korrelationen

\begin{tabular}{|c|c|c|c|c|c|c|c|c|c|c|c|}
\hline Variablen & Mittelwert & Std.abw. & $1 a$ & $1 b$ & 2 & 3 & 4 & 5 & 6 & 7 & 8 \\
\hline $\begin{array}{l}\text { WM-Punkte } \\
\text { Fahrer (1a) }\end{array}$ & 30,31 & 36,70 & & & & & & & & & \\
\hline $\begin{array}{l}\text { WM-Punkte } \\
\text { Konstrukteur (1b) }\end{array}$ & 61,69 & 69,35 & $0,94^{\star \star *}$ & & & & & & & & \\
\hline Fahrerqualität (2) & 58,73 & 68,43 & $0,77^{\star \star *}$ & $0,74^{\star \star \star}$ & & & & & & & \\
\hline $\begin{array}{l}\text { Leistung } \\
\text { Rennwagen (3) }\end{array}$ & $-42,92$ & 24,14 & $0,66^{\star * *}$ & $0,70^{\star \star \star}$ & $0,60^{\star \star \star}$ & & & & & & \\
\hline $\begin{array}{l}\text { Zuverlässigkeit } \\
\text { Rennwagen (4) }\end{array}$ & 77,78 & 15,13 & $0,53^{* * *}$ & $0,53^{* \star *}$ & $0,40^{\star \star *}$ & $0,35^{\star \star \star}$ & & & & & \\
\hline Teamdauer (5) & 19,00 & 16,03 & $0,67^{\star \star *}$ & $0,72^{\star \star \star}$ & $0,71^{\star \star \star}$ & $0,48^{\star \star \star}$ & $0,42^{\star \star \star}$ & & & & \\
\hline $\begin{array}{l}\text { Michelin/ } \\
\text { Bridgestone (6) }\end{array}$ & 0,44 & 0,50 & $-0,08$ & $-0,09$ & $-0,01$ & $-0,28^{\star \star \star}$ & $-0,12$ & 0,12 & & & \\
\hline Budget (7) & 204,62 & 97,13 & $0,56^{\star \star \star}$ & 0,60 *** & $0,60^{\star \star \star}$ & $0,68^{\star \star \star}$ & $0,33^{\star \star *}$ & $0,44^{* \star *}$ & $-0,29 \star \star$ & & \\
\hline $\begin{array}{l}\text { Kooperations- } \\
\text { dauer (8) }\end{array}$ & 9,25 & 15,57 & $0,63^{\star \star \star}$ & $0,66^{\star \star \star}$ & $0,70^{\star \star \star}$ & $0,47^{\star \star \star}$ & $0,42^{\star \star \star}$ & $0,78^{\star \star *}$ & $0,31 * *$ & $0,39^{\star \star *}$ & \\
\hline $\begin{array}{l}\text { Nicht integriert/ } \\
\text { integriert (9) }\end{array}$ & 0,38 & 0,49 & $0,35^{\star \star \star}$ & $0,36^{\star \star *}$ & $0,29^{\star \star \star}$ & $0,29 * \star \star$ & $0,21^{*}$ & 0,10 & $-0,23^{*}$ & $0,52^{\star \star \star}$ & $0,38^{\star \star \star}$ \\
\hline
\end{tabular}

Pearson Korrelationen, $n=104,{ }^{*} p<0,05 ;{ }^{* \star} p<0,01,{ }^{* \star *} p<0,001$

In Abschnitt 2.2.3 wurde bereits argumentiert, dass der Grad der vertikalen Integration einen direkten kausalen Einfluss auf die Höhe des dem Rennstall zur Verfügung stehenden Budgets und die Dauer der Kooperationsbeziehung zwischen Rennstall und Motorenlieferant ausübt.

Ein erster Gruppenvergleich zeigt, dass das durchschnittliche Budget eines integrierten Teams mit knapp 268 Mio. € über 100 Mio. € höher als das nicht-integrierter Teams liegt (t $=-6,089 ; \mathrm{p}<0,001)$. Auch mit einer durchschnittlichen Kooperationsdauer von knapp 17 Jahren - im Vergleich zu knapp 5 Jahren bei nicht-integrierten Teams - ist ein deutlicher Unterschied festzustellen ( $\mathrm{t}=-4,182 ; \mathrm{p}<0,001)$. Beide Gruppenunterschiede zeigen sich hoch signifikant. 
Die einzelnen Pfadkoeffizienten wurden mit Hilfe einer OLS-Schätzung ermittelt. Aufgrund der Unbekanntheit der Verteilungseigenschaften der unabhängigen Variablen in der Grundgesamtheit wurde neben dem standardmäßigen t-Test die Resampling-Methode des Bootstrappings zur statistischen Signifikanzüberprüfung genutzt (Efron, 1979; Leger et al., 1992). Beide Verfahren führten für alle Regressionskoeffizienten zu übereinstimmenden Schätzungen, so dass von einem effizienten Schätzergebnis ausgegangen werden kann.

Tabelle 2: Einfache OLS-Regression des vertikalen Integrationsgrads auf Budget und Kooperationsdauer

\begin{tabular}{lcc}
\hline Messwerte & Budget & Kooperationsdauer \\
\hline $\mathrm{R}^{2}$ & 0,267 & 0,146 \\
korr. $\mathrm{R}^{2}$ & 0,259 & 0,138 \\
F-Statistik & $37,08^{\star \star \star}$ & $17,49^{\star \star \star}$ \\
stand. Beta & $0,516^{\star \star \star}(16,85)$ & $0,383^{\star \star \star}(2,91)$ \\
Anzahl Fälle & 104 & 104 \\
\hline
\end{tabular}

${ }^{*} \mathrm{p}<0,05 ;{ }^{* \star} \mathrm{p}<0,01 ;{ }^{* \star \star} \mathrm{p}<0,001 ;$ Standardfehler in Klammern

Es zeigt sich, dass die Zusammenhänge zwischen vertikalen Integrationsgrad und Budget sowie Kooperationsdauer hoch signifikant auf einem Niveau von $\mathrm{p}<0,001$ sind. Der Grad der vertikalen Integration alleine erklärt bereits einen großen Teil der Varianz der Teambudgets $\left(R^{2}=0,27\right)$ und weist einen stark positiven Einfluss auf, womit Hypothese 6 Unterstützung findet. Vertikal integrierte Teams verfügen demnach über ein deutlich höheres Budget für den Rennbetrieb als nicht-integrierte Rennställe. ${ }^{22}$

Ebenso zeigt sich ein deutlich positiver signifikanter Einfluss auf die Kooperationsdauer zwischen Motorenlieferant und Rennstall, so dass auch Hypothese 7 bestätigt werden kann. Der erklärte Anteil der Varianz der Kooperationsdauer liegt dabei aber mit knapp 15\% deutlich niedriger als der des Teambudgets. Dies ist unter anderem darauf zurückzuführen, dass der Trend zu vertikaler Integration in der Formel 1 erst seit wenigen Jahren zu beobachten ist. So sind die Rennställe von Renault und Toyota erst seit dem Jahr 2002 integriert und insbesondere BMW und Honda gehen erst seit der Saison 2006 als integriertes Team an den Start. Der Zusammenhang zwischen Integration und Kooperationsdauer wird sich daher erst in einigen Jahren deutlicher in den Statistiken niederschlagen können. ${ }^{23}$

Auf der nächsten Stufe wurde ein Zusammenhang zwischen Budget und Kooperationsdauer sowie Fahrerqualität und Leistung und Zuverlässigkeit des Rennwagens postuliert. Dabei wurde davon ausgegangen, dass bei höherem Budget die Qualität des Fahrers steigt. Wie 
die einfache lineare Regressionsschätzung zeigt, werden über 35\% der Varianz der Fahrerqualität durch das Budget des Rennstalls bestimmt $(\mathrm{p}<0,001) .{ }^{24}$ Damit gilt auch Hypothese $4 \mathrm{a}$ als bestätigt.

Tabelle 3: OLS- Schätzung des Budgets auf Fahrerqualität

\begin{tabular}{lc}
\hline Messwerte & Fahrerqualität \\
\hline $\mathrm{R}^{2}$ & 0,359 \\
korr. $\mathrm{R}^{2}$ & 0,353 \\
F-Statistik & $57,21^{\star \star \star}$ \\
stand. Beta & $0,599^{\star \star \star}(0,056)$ \\
Anzahl Fälle & 104 \\
\hline
\end{tabular}

${ }^{\star} \mathrm{p}<0,05 ;{ }^{\star \star} \mathrm{p}<0,01 ;{ }^{\star \star \star} \mathrm{p}<0,001 ;$ Standardfehler in Klammern

Die Leistungsfähigkeit und Zuverlässigkeit eines Rennwagens ist nicht nur von dem zur Verfügung stehenden Budget abhängig, sondern wird auch positiv durch die Dauer der Zusammenarbeit zwischen Rennstall und Motorenlieferant geprägt. Modell 1 der folgenden multiplen Regression analysiert diesen Zusammenhang (Tabelle 4). Um auf mögliche Multikollinearität zu kontrollieren, wurde der „Variance Inflation Factor“ berechnet. In beiden Regressionen lagen die höchsten Werte bei 1,18. Die Werte sind somit in einem akzeptablen Bereich, so dass alle Variablen beibehalten werden konnten (Hair et al., 1998). Als Kontrollvariable wird jeweils in Modell 2 die Teamdauer eingeführt, da diese ebenfalls einen Einfluss auf die Entwicklungsfähigkeit der Teams ausüben kann.

Die Regression ohne Kontrollvariable verdeutlicht, dass sowohl Budget als auch Kooperationsdauer einen deutlichen und signifikanten Einfluss auf die Leistungsfähigkeit als auch auf die Zuverlässigkeit des Rennwagens ausüben. Dabei wird im Fall der Leistungsfähigkeit durch die beiden unabhängigen Variablen 52\% der Varianz erklärt, wobei das Budget einen deutlich stärkeren Einfluss auf die abhängige Variable ausübt $(0,587)$ als die Kooperationsdauer $(0,243)$. Interessanterweise dreht sich diese Reihenfolge bei der Erklärung der Zuverlässigkeit des Rennwagens um. Hier werden insgesamt 21\% der Varianz erklärt, wobei die Kooperationsdauer einen deutlich stärkeren Einfluss $(0,348)$ auf die Zuverlässigkeit des Wagens ausübt, als das Budget $(0,190)$. Dies ist damit zu begründen, dass sich die Leistung eines Rennwagens auch durch separate Entwicklung des Motors steigern lässt, wobei die Zuverlässigkeit eines Rennwagens davon abhängt, wie alle Komponenten aufeinander 
abgestimmt werden. Da gerade die Kooperationsdauer auf eine bessere Abstimmung von Motor und Chassis hinweist, ist der stärkere Einfluss eine logische Konsequenz.

Unter Einbeziehung der Kontrollvariablen konnte die Erklärungsgüte der Regressionen nicht deutlich verbessert werden. Hinzu kommt, dass sich der Einfluss der Kooperationsdauer nicht mehr signifikant darstellt. Dies kann auf Multikollinearität zwischen Teamdauer und Kooperationsdauer zurückzuführen sein, die mit einem Korrelationskoeffizienten von 0,788 und einem VIF von 2,68 nicht auszuschließen ist. Damit kann Modell 1 als das überlegene Modell angesehen werden. Hypothesen 4b, 4c, 5a und 5b können somit als bestätigt gelten.

Tabelle 4: Multiple OLS-Regression auf Leistung und Zuverlässigkeit des Rennwagens

\begin{tabular}{|c|c|c|c|c|}
\hline \multirow[b]{2}{*}{ Variablen } & \multicolumn{2}{|c|}{ Leistung Rennwagen } & \multicolumn{2}{|c|}{ Zuverlässigkeit Rennwagen } \\
\hline & Modell 1 & Modell 2 & Modell 1 & Modell 2 \\
\hline Budget & $0,587^{\star \star \star}(0,019)$ & $0,574^{\star \star \star}(0,019)$ & $0,190 *(0,015)$ & $0,162^{*}(0,015)$ \\
\hline Koordinationsdauer & $0,243^{\star \star}(0,117)$ & $0,188(0,172)$ & $0,348^{\star \star \star}(0,094)$ & $0,222(0,137)$ \\
\hline Teamdauer & & $0,077(0,171)$ & & $0,177(0,136)$ \\
\hline $\mathrm{R}^{2}$ & 0,515 & 0,503 & 0,210 & 0,222 \\
\hline korr. $\mathrm{R}^{2}$ & 0,506 & 0,518 & 0,194 & 0,198 \\
\hline F-Statistik & $53,69 * \star \star$ & $35,76^{\star \star \star}$ & $13,425^{\star \star \star}$ & $9,496 * \star \star$ \\
\hline Anzahl Fälle & 104 & 104 & 104 & 104 \\
\hline
\end{tabular}

${ }^{*} \mathrm{p}<0,05 ;{ }^{* *} \mathrm{p}<0,01 ;{ }^{* * *} \mathrm{p}<0,001 ;$ Standardfehler in Klammern

Offen blieb bisher der Zusammenhang zwischen den Mediatorvariablen und der eigentlichen Erfolgsvariable WM-Punktzahl. Hier wurde ein Einfluss über Fahrerqualität, Leistung und Zuverlässigkeit des Rennwagens vermutet, den es im Folgenden zu analysieren gilt. Alle Variablen wurden in einer einfachen Regression daraufhin überprüft, ob ein linearer Zusammenhang zur WM-Punktzahl besteht, oder ob die Erklärungsgüte der Regression durch einen nicht-linearen Zusammenhang gesteigert werden kann. Bis auf den Zusammenhang zwischen WM-Punktzahl und Leistungsfähigkeit des Rennwagens bot die lineare Einfachregression die besten Schätzwerte.

Im Falle der Leistung des Rennwagens konnte ein Strukturbruch festgestellt werden. Erst ab einer absoluten Abweichung der schnellsten Rundenzeiten von insgesamt 45\% oder weniger hatte diese einen Einfluss auf die WM-Punktzahl. Lag die Abweichung darüber, hatte 
sie keinen Einfluss auf die abhängige Variable. Die lineare Regression über alle Leistungsklassen ergibt einen erklärten Varianzanteil der WM-Punktzahl von 43\%, betrachtet man nur die Leistungsklassen mit einer absoluten Abweichung unter 45\%, erhöht sich die Erklärungsgüte auf 65\%. Dies kann damit begründet werden, dass es einen Schwellenwert der Leistungsfähigkeit eines Rennwagens gibt, der auf jeden Fall überschritten werden muss, um in der Konkurrenz um die Punkteplätze in den einzelnen Grand Prix-Rennen mitzufahren. Da ein Ausschluss der angegebenen Datensätze in der multiplen Regression jedoch zu Verzerrungen in den unabhängigen Variablen Fahrerqualität und Zuverlässigkeit führen würde, wurden alle Datensätze weiterhin in die Analyse einbezogen. Dabei wurden drei verschiedene Modelle geschätzt. In Modell 1 wurde ausschließlich der Einfluss des Fahrers auf die WM-Punktzahl betrachtet. Modell 2 erweitert die Analyse um die Einflussfaktoren des Rennwagens, während Modell 3 die Kontrollvariable Reifenhersteller in die Auswertung einbezieht.

Unter ausschließlicher Betrachtung der Fahrerqualität in Modell 1 zeigt sich ein stark signifikanter Zusammenhang zur WM-Punktzahl Fahrer, der bereits einen hohen Erklärungsanteil enthält $\left(R^{2}=0,6\right)$. Die in der Rennpraxis stets hervorgehobene besondere Bedeutung des Fahrers für den Rennerfolg eines Teams scheint sich hier zu bestätigen. Auch unter Einbeziehung der Erfolgskomponenten des Rennwagens in Modell 2, der den Erklärungsgehalt des WM-Erfolgs erhöht $\left(R^{2}=0,7\right)$, bleibt die Fahrerqualität der Faktor mit dem höchsten Erfolgseinfluss (0,525). Aber auch die Leistungsfähigkeit und die Zuverlässigkeit des Rennwagens üben mit 0,231 respektive 0,259 noch einen starken signifikanten Einfluss auf das Renngeschehen aus. Dagegen zeigt sich in Modell 3 unter Einbeziehung des Reifenherstellers für diesen kein signifikanter Erfolgseinfluss. Der VIF liegt bei allen Variablen unter 1,8 und damit auch hier in einem akzeptablen Bereich, der keine Probleme durch Multikollinearität vermuten lässt. ${ }^{25}$ 
Tabelle 5: Ergebnisse der OLS Schätzungen mit WM-Punktzahl als abhängiger Variable

\begin{tabular}{|c|c|c|c|c|}
\hline & & WM-Punkte Fahre & & WM-Punkte Konstrukteur \\
\hline Variablen & Modell 1 & Modell 2 & Modell 3 & Modell 2 \\
\hline Fahrerqualität & $0,772^{\star \star \star}(0,03)$ & $0,525^{\star \star *}(0,04)$ & $0,518^{\star \star \star}(0,039)$ & $0,435^{\star \star \star}(0,072)$ \\
\hline Leistung Rennwagen & & $0,259^{\star \star \star}(0,106)$ & $0,270^{\star \star \star}(0,112)$ & $0,362^{\star \star \star}(0,201)$ \\
\hline Zuverlässigkeit Rennwagen & & $0,231^{\star \star \star}(0,147)$ & $0,233^{\star \star \star}(0,148)$ & $0,225^{\star \star \star}(0,279)$ \\
\hline Reifenhersteller & & & $0,026(4,333)$ & \\
\hline $\mathrm{R}^{2}$ & 0,60 & 0,70 & 0,70 & 0,70 \\
\hline korr. $\mathrm{R}^{2}$ & 0,59 & 0,69 & 0,69 & 0,69 \\
\hline F-Statistik & $150,91^{* * *}$ & $76,96 * \star *$ & $57,30^{\star \star \star}$ & $76,00^{\star \star \star}$ \\
\hline Anzahl Fälle & 104 & 104 & 104 & 104 \\
\hline
\end{tabular}

${ }^{*} \mathrm{p}<0,05 ;{ }^{* \star} \mathrm{p}<0,01 ;{ }^{* \star *} \mathrm{p}<0,001 ;$ Standardfehler in Klammern

Da sich die Erklärungsgüte von Modell 3 im Vergleich zu Modell 2 nicht verbessert, können die Zusammenhänge in Letzterem als die beste Schätzung angesehen werden. Ähnliche Ergebnisse zeigen sich bei einer Analyse des Einflusses der Erfolgsfaktoren auf die WMPunktzahl Konstrukteur. Hier zeigt sich ein gleich hoher Erklärungsgehalt $\left(R^{2}=0,7\right)$, wobei sich die Relation der Erfolgseinflüsse leicht verschiebt. So verstärkt sich bei Betrachtung der WM-Punktzahl Konstrukteur der Einfluss der Leistungsfähigkeit des Rennwagens im Vergleich zur Fahrerqualität und Zuverlässigkeit des Rennwagens. Insgesamt können die Hypothesen 1 bis 3 somit bestätigt werden. ${ }^{26}$

Die dargestellten Ergebnisse könnten möglicherweise durch die Ausnahmestellung des Ferrari-Teams mit seinem siebenmaligen Weltmeister Michael Schumacher zu Gunsten des Einflusses der Fahrerqualität verzerrt sein. Eine OLS-Schätzung unter Ausschluss der Rennergebnisse von Michael Schumacher zeigt, dass der Einfluss der Fahrerqualität tatsächlich überschätzt wurde. In einem adaptierten Modell 2 auf WM-Punktzahl Fahrer zeigt sich, dass mit einem standardisierten Beta von 0,396 die Fahrerqualität zwar immer noch die wichtigste Erfolgskomponente darstellt, die Leistung $(0,387)$ und die Zuverlässigkeit des Rennwagens $(0,251)$ aber im Vergleich deutlich mehr Bedeutung gewinnen. ${ }^{27}$ An den grundsätzlichen Aussagen zum Erfolgseinfluss der drei Variablen ändert sich hingegen nichts. Abbildung 2 gibt abschließend einen Überblick über die statistischen Zusammenhänge im gesamten Pfadmodell (Modell 2). 


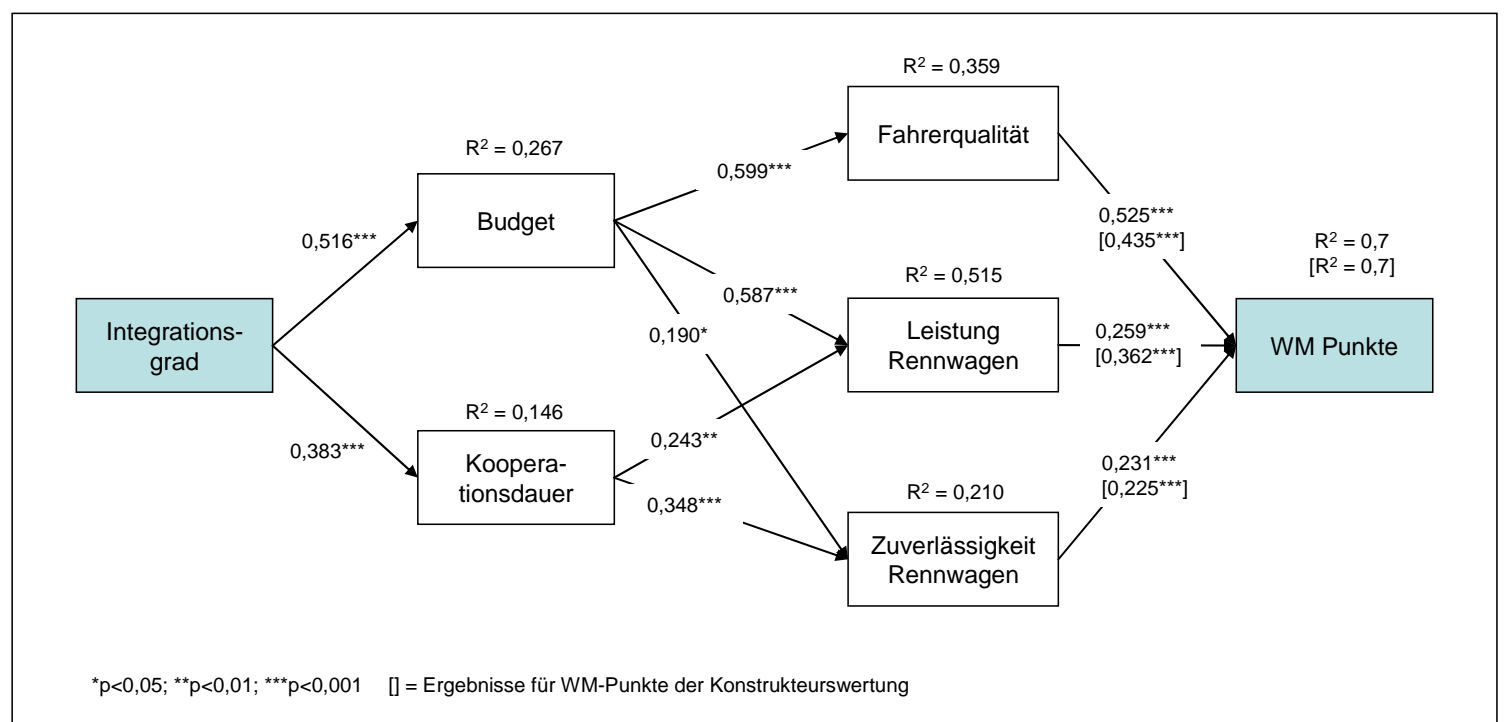

\section{Diskussion und Ausblick}

Empirische Felduntersuchungen beschäftigen sich vielfach allein mit der Frage, welche Transaktionstypen innerhalb eines Unternehmens durchgeführt werden sollten. Dabei wird üblicherweise auf das spezifische Transaktionsumfeld abgestellt, in dem die vertikale Integration stattfindet. Solche Analysen haben vor allem den Nachteil, dass Rückschlüsse auf die unmittelbare Erfolgswirkung der vertikalen Integration auf Unternehmensebene nur schwer möglich sind. Somit kann vielfach nicht eindeutig bestimmt werden, ob beispielsweise ein höherer Unternehmensgewinn oder ein höheres Investitionsniveau direkt auf die Veränderung des Integrationsgrads zurückzuführen oder durch andere exogene Faktoren bedingt ist. Genau an diesem Defizit setzt die vorliegende empirische Untersuchung an. Primärer Forschungsschwerpunkt ist die Frage, ob die Erhöhung der Leistungstiefe beziehungsweise des vertikalen Integrationsgrads zentraler Wertschöpfungsaktivitäten unter bestimmten Marktgegebenheiten zu einer Erhöhung der Investitionsneigung und zu einer Verbesserung des Unternehmenserfolgs führt. Um die unmittelbare Wirkung des Integrationsgrads auf Unternehmensebene analysieren zu können, wird die Forschungsfrage in der vorliegenden Arbeit im spezifischen Umfeld des Formel 1-Rennsports bearbeitet.

Das Transaktionsumfeld in der Formel 1 ist durch explizite Wettbewerbsregeln stark kontrolliert und relativ stabil. Bedingt durch die andauernde, intensive Entwicklungsarbeit in den vergangenen Jahrzehnten sind isolierte Leistungssteigerungen im Motoren- bzw. Chassisbereich sehr kostenintensiv. Somit hängt der Teamerfolg vor allem von der koordinierten 
Erst- und Weiterentwicklung des Rennwagens durch Rennstall und Motorenhersteller ab. Zentrale Probleme der Zusammenarbeit resultieren insbesondere daraus, dass die Transaktionspartner sowohl transaktionsspezifische Investitionen tätigen müssen und gleichzeitig die Entwicklungsanstrengungen des jeweils anderen Kooperationspartners nur unzureichend beobachten können. Im Rahmen der empirischen Analyse konnte zunächst gezeigt werden, dass die vertikale Vorwärtsintegration von Rennställen durch große Automobilkonzerne den Teamerfolg erhöht. Somit bestätigt die empirische Analyse modelltheoretische Ergebnisse der Transaktionskosten- und der Prinzipal-Agenten Theorie.

Die statistische Pfadanalyse fördert darüber hinaus weitere Einblicke hinsichtlich der spezifischen Wirkung des Integrationsgrads auf Unternehmensebene zutage. Die Analyse verdeutlicht vor allem, dass vertikal integrierte Teams deutlich höhere Budgets als nichtintegrierte Teams aufweisen. Dieser Umstand deutet darauf hin, dass Unterinvestitionsprobleme, im betrachteten Transaktionsumfeld mit hoher Spezifität und gleichzeitigen Messproblemen hinsichtlich der individuellen Leistungsbeiträge, innerhalb der hierarchischen Organisation tendenziell besser gelöst werden. Bedingt durch die Erhöhung des Investitionsvolumens können integrierte Teams leistungsfähigere Fahrer an sich binden sowie gleichzeitig die Leistung und Zuverlässigkeit der eingesetzten Rennwagen erhöhen. Diese Faktoren wirken ihrerseits wiederum signifikant auf die Erfolgswahrscheinlichkeit bei Grand Prix-Rennen. Neben einer verbesserten Anreizsetzung erhöht die vertikale Integration die Kooperationsdauer zwischen den Transaktionspartnern, so dass sich die Leistungsfähigkeit des Rennwagens und somit mittelbar der Erfolg des Formel 1-Teams erhöht.

Der vorliegende Beitrag hat die Auswirkungen der vertikalen Integration insbesondere aus Sicht des Unterinvestitionsproblems empirisch beleuchtet. Offen bleibt, ob die positive Erfolgswirkung auch dann noch beobachtet werden kann, wenn mögliche Nachteile der vertikalen Integration betrachtet werden, die in der Formel 1 eine untergeordnete Rolle spielen. So geht man davon aus, dass die vertikale Integration strategische Kosten durch eingeschränkte Flexibilität sowie durch steigende Marktaustrittsbarrieren auslösen kann. Darüber hinaus kann eine Erhöhung der Produktionskosten resultieren, wenn Kapazitäten suboptimal genutzt werden oder die Produktionsmenge reduziert wird (Stigler, 1968; Cremer, 1980; Williamson, 1967, 1975, 1985; Harrigan, 1985). Folgestudien sollten daher die Auswirkungen der vertikalen Integration in Märkten untersuchen, in denen genau diese Prob- 
lemfelder auftreten, um einen möglichen Interaktionseffekt mit dem Unterinvestitionsproblem zu analysieren.

\section{Anmerkungen}

1 Vgl. hierzu die sehr guten Überblicksarbeiten von Mahoney (1992), Picot und Franck (1993) sowie Shelanski und Klein (1995).

2 Alle Angaben in diesem Abschnitt beziehen sich auf das Jahr 2006.

3 Der Technologietransfer von Innovationen aus dem Motorsport in die Serienfertigung spielt nur eine untergeordnete Rolle. Die Auswirkungen auf das Markenimage sind dagegen nachgewiesen. So stieg die Markensympathie für BMW oder Mercedes durch ihr Formel 1-Engagement um 20 - 30\% (vgl. Icon brand navigation, 2000).

4 Vgl.www.fia.com/resources/documents/149730155_2007_F1_SPORTING_REGULATIONS.pdf sowie www.fia.com/resources/documents/1875416810_2007_F1_TECHNICAL_REGULATIONS.pdf

5 In der Formel 1 können ganz unterschiedliche Formen von Partnerschaften unterschieden werden. Während klassische Zulieferer Güter und Dienstleistungen gegen direkte Bezahlung zur Verfügung stellen, beziehen sich technische Partnerschaften auf die direkte Bereitstellung von Gütern und Dienstleistungen im Austausch mit Marketingmöglichkeiten. Vgl. Jenkins et al. (2005), S. 85f.

6 Vgl. FIA Formula One World Championship Regulations, www.fia.com/sport/Regulations/f1regs.html.

7 Diese wird von der FIA auf Antrag im Wesentlichen an solche Fahrer vergeben, die bereits eine Grade A competition licence haben und zudem in einer der unteren Rennklassen (Formel 3 oder GP2 Serie) konstant hervorragende Leistungen gezeigt haben. Vgl. FIA Formula One World Championship Regulations Appendix L, www.fia.com/resources/documents/1797101136_Appendix_L_a.pdf

8 Die Vorteile der vertikalen Integration in Transaktionsbeziehungen, die durch hohe Spezifität gekennzeichnet sind, findet in der empirischen Literatur weite Bestätigung. Zudem konnte gezeigt werden, dass die Investitionsneigung bei vertikaler Integration in diesen Fällen deutlich steigt. Vgl. Williamson (1971), Mahoney (1992) sowie Ciliberto (2006).

9 Einen guten Überblick über empirische Arbeiten, die die Tendenz zur vertikalen Integration bei Vorliegen von Messproblemen bestätigen, gibt Mahoney (1992). Zu den Auswirkungen von Gruppendruck und gegenseitiger Kontrolle vgl. insbesondere Kandel/Lazear (1992) sowie Knez/Simester (2001).

10 Es kann argumentiert werden, dass höhere Budgets integrierter Teams zum Teil auf begrenzte finanzielle Möglichkeiten nicht-integrierter Teams zurückzuführen sind. In diesem Fall läge das Problem der optimalen Organisation unter begrenzter Verschuldbarkeit vor. Rennställe finanzieren sich überwiegend über Sponsorengelder und materielle Leistungen aus technischen und unternehmerischen Partnerschaften (Jenkins, 2005). Somit stellt sich die Frage, warum nicht-integrierte Rennställe für externe Sponsoren oder Partner weniger attraktiv sind als integrierte. Doch selbst dann, wenn man die Tatsache beschränkter Finanzmittel als gegeben ansieht und annimmt, dass Rennställe keinen Zugang zu anderen externen Kapitalquellen haben, stellt sich die Frage, warum große Automobilhersteller die Option der vertikalen Vorwärtsintegration wählen anstatt den Rennställen entsprechendes Kapital zur Verfügung stellen. Wir gehen daher davon aus, dass die Investitionsneigung entscheidend durch die beschriebene Hold-Up- und Messproblematik beeinflusst wird.

11 In den Jahren vor 2002 traten nur Ferrari und Jaguar als integrierte Teams an. Erst mit dem Jahr 2002 und dem Einstieg von Renault und Toyota in die Rennserie konnte die Tendenz zur Integration beobachtet werden, die zu einer ausreichend großen Datengrundlage führte.

12 Vgl. FIA Formula One World Championship Regulations, www.fia.com/sport/Regulations/f1regs.html.

13 Anzahl der Teams pro Saison: 2002 (11), 2003 (10), 2004 (10), 2005 (10) und 2006 (11).

14 Es wurden die Datenbanken des Formel 1 Informationsdienstes F1Total.com, die Jahrbücher „Grand Prix 200X - Formel 1 live miterlebt“ von Willy Knupp im Auftrag von RTL sowie die offiziellen Datenbanken der Formel One Management Limited für die Datenerhebung genutzt (www.formula1.com). 
15 Um Datenverzerrungen durch die Umstellung des Punktesystems im Jahr 2003 zu vermeiden, wurden alle Rennergebnisse der Jahre 2000 bis 2002 der einzelnen Fahrer in das neue Punktesystem von 2003 überführt. Ein alternatives Erfolgsmaß stellt die Endplatzierung eines Fahrers bzw. Teams in jedem Grand Prix bzw. in einer Saison dar (siehe FN 25). Für die Außenwirkung der Rennställe im Markt ist dieses Maß sicherlich von höherer Bedeutung als die reinen WM-Punkte, denn der Gewinn der WM mit 90 ist wichtiger als ein zweiter Platz mit 95 Punkten. Die WM-Punkte zeigen aber die relative Stärke eines Teams im Vergleich des Wettbewerbsfeldes. Da in jedem Grand Prix gleich viele Punkte gewonnen werden können, ist die Vergleichbarkeit der Rennställe untereinander über mehrere Jahre so besser möglich.

16 So fuhr Michael Schumacher in den betrachteten Rennjahren ausschließlich für Ferrari während beispielsweise Juan Pablo Montoya zwischen BMW Williams und McLaren Mercedes wechselte. Giancarlo Fisichella wechselte in den Rennjahren 2002-2006 sogar dreimal den Rennstall, von Jordan über Sauber zu Renault. Um die Trennschärfe zwischen Fahrerqualität und Leistungsfähigkeit des Rennwagens zu verbessern, wurden zwei alternative Qualitätsmaße berechnet und auf die WM-Punktzahl regressiert. Zum einen wurden die relativen Platzierungen eines Fahrers in den letzten beiden Formel 1 Saisons sowie den letzten beiden Saisons in einer anderen Rennklasse (z.B. Formel 3000) genutzt. Damit wird die Qualität des Fahrers über verschiedene Rennwagen gemessen und der Einfluss der Rennwagenqualität minimiert. Die OLS-Ergebnisse zeigen, dass die grundsätzlichen Zusammenhänge auch für dieses Qualitätsmaß weiter gelten. Zum anderen haben wir die Rangwerte von Stadelmann/Eichenberger (2007) entwickelten historischen Fahrerrankings genutzt. Diese haben in einem ökonometrischen Modell gezielt den Einfluss des Rennwagens auf die Fahrerqualität herausgerechnet. Leider liegen hier nur Werte zu 19 von 38 Fahrern unserer Betrachtung vor. Die verbleibenden Fahrer haben wir daher über die durchschnittliche Fahrerqualität aller Fahrer berechnet. In einer OLS-Regression auf die WM-Punktzahl bestätigt sich auch hier der generelle Zusammenhang zwischen Fahrerqualität und WM-Erfolg. Die entsprechenden Schätzungen können bei Bedarf bei den Autoren angefordert werden.

17 Wurde die schnellste Rundenzeit eines Fahrers durch einen technischen Defekt des Wagens beeinträchtigt, so wurde diese nicht in die Bewertung aufgenommen, sondern durch die durchschnittliche Abweichung über alle verbleibenden Rennen ersetzt.

18 Hierbei handelt es sich um die Jahrbücher „Grand Prix 200X - Formel 1 live miterlebt“ von Willy Knupp im Auftrag von RTL.

19 Die Rennställe von Honda, Renault und Toyota gehören zu 100\% den gleichnamigen Automobilkonzernen. BMW hält 85\% am Eigenkapital des BMW Sauber F1 Teams, Fiat ist zu 56\% am Ferrari-Team beteiligt. Dass dieses Team trotzdem als integriert gilt, hängt mit der 34 \%-igen Beteiligung der Medio Banca zusammen, die sich als reiner Finanzinvestor sieht und nicht in die Teamgeschäfte eingreift.

20 Varianzhomogenität ist auf einem Signifikanzniveau von $1 \%$ gegeben.

21 Die Schätzung eines regressionsbasierten Totalmodells zeigt, dass wider Erwarten weder Integrationsgrad noch Budget oder Kooperationsdauer einen signifikanten Einfluss auf die WM-Punktzahl ausüben. Zudem ergibt sich das Problem hoher Multikollinearität. Dies weist auf starke Mediatorbeziehungen zwischen den einzelnen unabhängigen Variablen hin, die sich auch aus der inhaltlichen Entwicklung der Hypothesen ergeben. Somit ist die Schätzung eines Pfadmodells, also die Schätzung einzelner Regressionen, an dieser Stelle vorzuziehen. Die Nutzung kovarianzbasierter Verfahren (Amos, LISREL) ist aufgrund der Datenbasis und den für diese Verfahren zugrundeliegenden Verteilungsannahmen nicht möglich. Varianzbasierte Verfahren (PLS) können dagegen gut eingesetzt werden, ergeben aber bei Variablen, die nicht über Konstrukte gemessen werden, keine Unterschiede zu normalen multiplen Regressionsanalysen. Zur Überprüfung wurde das Pfadmodell auch über PLS berechnet. Sowohl die Pfadkoeffizienten als auch die Signifikanzniveaus entsprechen denen der Einzelregressionen.

22 Es besteht die Möglichkeit, dass nicht der Integrationsgrad die Höhe des Teambudgets beeinflusst, sondern ein umgekehrter kausaler Zusammenhang vorliegt, dass also das Budget den Integrationsgrad bestimmt. Um diesen Zusammenhang auszuschließen wurden Mittelwertvergleiche zwischen den Budgets integrierter Teams vor Integration mit denen nicht-integrierter Teams durchgeführt. Dabei zeigen sich weder für einen längeren Betrachtungszeitraum von 1999-2005 noch für eine separate Betrachtung des Jahres 2005 signifikante Unterschiede. Es ist daher davon auszugehen, dass der Integrationsgrad nicht durch das Teambudget beschrieben werden kann. 
23 Da das Ferrari-Team bereits seit ihrem Eintritt in die Formel 1 vor knapp 60 Jahren als integrierter Rennstall auftritt, könnten sich Verzerrungen hinsichtlich Kooperationsdauer ergeben. Eine Analyse der Gruppenunterschiede ohne das Ferrari-Team zeigt, dass sich integrierte und nicht-integrierte Teams hinsichtlich Kooperationsdauer dann nicht mehr signifikant unterscheiden. Da die Mehrzahl der integrierten Teams jedoch erst im Beobachtungszeitraum von 2002 bis 2006 entstanden, kann bei diesen noch kein Einfluss auf die Kooperationsdauer nachgewiesen werden. Es ist jedoch für die Zukunft davon auszugehen, dass sich durch Integration die Kooperationsdauer verlängert. Diese Hypothese kann aber erst in einigen Jahren gesichert überprüft werden. Die generellen Aussagen zu Gruppenunterschieden hinsichtlich des Budgets verändern sich bei Ausschluss des Ferrari-Teams nicht.

24 Die Möglichkeit abnehmender Grenznutzen des Budgets wurde durch geeignete nicht-lineare Regressionen überprüft. Es hat sich gezeigt, dass dadurch die Anpassungsgüte im Vergleich zur linearen Regression nicht verbessert werden konnte, so dass davon auszugehen ist, dass im Bereich der Budgetwerte, die derzeit vorliegen, ein linearer Zusammenhang zwischen unabhängiger und abhängiger Variable besteht.

25 Auch wenn der VIF-Wert nicht auf Multikollinearität hinweist, haben wir in einer weiteren Analyse neben dem Budget und der Kooperationsdauer auch die Fahrerqualität auf die Leistungsfähigkeit des Rennwagens für jede WM separat regressiert. In keinem Jahr ergibt sich hier ein signifikanter Einfluss der Fahrerqualität auf die Leistungsfähigkeit des Rennwagens.

26 Die aufgezeigten Resultate für die abhängige Variable WM-Punkte gelten in ihrer Tendenz auch für das alternative Erfolgsmaß WM-Platzierung. Die ordinal logistische Regression für die WM-Platzierung Fahrer zeigt bei gegebener Modellgüte eine gute Anpassung (Nagelkerkes Pseudo $\mathrm{R}^{2}=0,753$ ). Die Korrelationskoeffizienten sind signifikant und bestätigen die postulierten Zusammenhänge (Fahrerqualität: -0,21***; Leistungsfähigkeit: -0,07***, Zuverlässigkeit: $-0,05^{* * *}$ ).

$27 \mathrm{R}^{2}=0,667$; alle Werte sind signifikant auf einem Niveau von $\mathrm{p}<0,001$.

\section{Literaturverzeichnis}

Alchian, Armen A./Demsetz, Harold (1972), Production, Information Costs, and Economic Organization, in: American Economic Review, Vol. 62, S. 777-795.

Ancona, Deborah G./Caldwell, David F. (1992), Bridging the boundary: External activity and performance in organizational teams, in: Administrative Science Quarterly, Vol. 37, S. 634-665.

Barney, Jay B. (1991), Firm resources and sustained competitive advantage, in: Journal of Management, Vol. 17, S. 99-120.

Chatterjee, Sayan (1991), Gains in vertical acquisitions and market power: Theory and evidence, in: Academy of Management Journal, Vol. 34, S. 436-448.

Ciliberto, Federico (2006), Does Organizational Form Affect Investment Decisions?, in: Journal of Industrial Economics, Vol. 54, S. 63-93.

Coase, Ronald H. (1937), The Nature of the Firm, in: Economica, Vol. 4, S. 386-405.

Cremer, Jacques (1980), A Partial Theory of the Optimal Organization of a Bureaucracy, in: Bell Journal of Economics, Vol. 11, S. 683-693.

D'Aveni, Richard A./Ravenscraft, David J. (1994), Economies of Integration versus Bureaucracy Costs: Does Vertical Integration Improve Performance?, in: Academy of Management Journal, Vol. 37, S. 11671206.

Efron, Bradley (1979), Bootstrap Methods: Another Look at the Jackknife, in: Annals of Statistics, Vol. 7, S. 1-26.

Eisenhardt, Kathleen M. (1989) Making Fast Strategic Decisions in High-Velocity Environments, in: Academy of Management Journal, Vol. 32, S. 543-576.

Grant, Robert M. (1991), The resource-based theory of competitive advantage, in: California Management Review, Vol. 33, S. 114-135.

Grossman, Sanford J./Hart, Oliver D. (1986), The costs and benefits of ownership: A theory of vertical and lateral integration, in: Journal of Political Economy, Vol. 94, S. 691-719.

Hair, Joseph F./Anderson, Rolph E./Tatham, Ronald L./Black, William C. (1998), Multivariate Data Analysis, New York et al.

Harrigan, Kathryn R. (1985), Strategic Flexibility, Lexington. 
Harrigan, Kathryn R. (1986), Matching Vertical Integration Strategies to Competitive Conditions, in: Strategic Management Journal, Vol. 7, S. 535-555.

Hart, Oliver/John Moore (1990), Property Rights and the Nature of the Firm, in: Journal of Political Economy, Vol. 98, S. 1119-1158.

Hoegl, Martin/Gemünden, Hans G. (2001), Teamwork Quality and the Success of Innovative Projects: A theoretical concept and Empirical evidence, in: Organization Science, Vol. 12, S. 435-449.

Hoegl, Martin/Weinkauf, Katharina/Gemuenden, Hans Georg (2004) Interteam Coordination, Project Commitment, and Teamwork in Multiteam R\&D Projects: A Longitudinal Study, in: Organization Science, Vol. 15, S. 38-55.

Holmström, Bengt (1982), Moral hazard in Teams, in: Bell Journal of Economics, Vol. 13, S. 324-340.

Icon brand navigation (2000), Vernetzung 2000: Formel 1: Integrierte Markenkommunikation im Motorsport. Studie der DSF Deutsches SportFernsehen GmbH.

Jenkins, Mark (2004), Innovative management: superior performance in changing times, in: European Business Journal, Vol. 16, S. 10-19.

Jenkins, Mark/Paternak, Ken/West,Richard (2005), Performance at the Limit - Business Lessons from Formula 1 Motor Racing, Cambridge.

Joskow, Paul J. (1988), Asset Specificity and the Structure of Vertical Relationships: Empirical Evidence, in: Journal of Law, Economics, and Organization, Vol. 4, S. 95-117.

Kandel, Eugene/Lazear, Edward P. (1992) Peer Pressure and Partnerships, in: Journal of Political Economy, Vol. 100, S. 801-817.

Kerkvliet, Joe (1991), Efficiency and Vertical Integration: The Case of Mine-Mouth Electric Generating Plants, in: Journal of Industrial Economics, Vol. 39, S. 467-482.

Klein, Benjamin/Crawford, Robert G./Alchian, Armen A. (1978), Vertical Integration, Appropriable Rents, and the Competitive Contracting Process, in: Journal of Law and Economics, Vol. 21, S. 297-326.

Klein, Howard J./Mulvey, Paul W. (1995), Two Investigations of the Relationships among Group Goals, Goal Commitment, Cohesion, and Performance, in: Organizational Behavior \& Human Decision Processes, Vol. 61, S. 44-53.

Knez, Marc/Simester, Duncan (2001), Firm-Wide Incentives and Mutual Monitoring at Continental Airlines, in: Journal of Labor Economics, Vol. 19, S. 743-772.

Knupp, Willy (2002), Grand Prix 2002 live miterlebt - Formel-1-Weltmeisterschaft, Düsseldorf.

Knupp, Willy (2003), Grand Prix 2003 live miterlebt - Formel-1-Weltmeisterschaft, Düsseldorf.

Knupp, Willy (2004), Grand Prix 2004 live miterlebt - Formel-1-Weltmeisterschaft, Düsseldorf.

Knupp, Willy (2005), Grand Prix 2005 live miterlebt - Formel-1-Weltmeisterschaft, Düsseldorf.

Knupp, Willy (2006), Grand Prix 2006 live miterlebt - RTL-Formel-1, Düsseldorf.

Leger, Christian/Politis, Dimitris N./Romano, Joseph P. (1992), Bootstrap Technology and Applications, in: Technometrics, Vol. 34, S. 378-398.

Leiblein, Michael J./Reuer, Jeffrey J./Dalsace, Frédéric (2002), Do Make or Buy Decisions Matter? The Influence of Organizational Governance on Technological Performance, in: Strategic Management Journal, Vol. 23, S. 817-833.

Mahoney, Joseph T. (1992), The Choice of Organizational Form: Vertical Financial Ownership versus other Methods of Vertical Integration, in: Strategic Management Journal, Vol. 13, S. 559-584.

McDonough, Edward F. (2000), An Investigation of Factors Contributing to the Success of Cross-Functional Teams, in: Journal of Product Innovation Management, Vol. 17, S. 221-235.

Penrose, Edith T. (1959). The Theory of the Growth of the Firm. New York.

Picot, Arnold/Franck, Egon (1993), Vertikale Integration, in: Hauschildt, Jürgen/Grün, Oskar (Hrsg.), Ergebnisse empirischer betriebswirtschaftlicher Forschung, Stuttgart, S. 179-214.

Poppo, Laura/Zenger, Todd (1998), Testing alternative theories of the firm: Transaction cost, knowledgebased, and measurement, in: Strategic Management Journal, Vol. 19, S. 853-877.

Renner, Hermann (2005), Ein teures Rennvergnügen - was kostet die Formel 1, Frankfurter Allgemeine Sonntagszeitung, 14.8.2005, Nr. 32, S. 21.

Shelanski, Howard A./Klein, Peter G. (1995), Empirical Research in Transaction Cost Economics: A Review and Assessment, in: Journal of Law, Economics \& Organization; Vol. 11, S. 335-361.

Simon, Herbert A. (1978), Rationality as process and as product of thought, in: American Economic Review Proceedings, Vol. 68, S. 1-16.

Spiller, Pablo T. (1985), On Vertical Mergers, in: Journal of Law, Economics, \& Organization, Vol. 1, S. 285312.

Stadelmann, David/ Eichenberger, Reiner (2007), Wer ist der beste Fahrer in der Formel 1? Eine ökonometrische Analyse. Working Paper Universität Fribourg.

Stigler, George J. (1968), The Organization of Industry, Homewood. 
Wernerfelt, Birger (1984), A resource-based view of the firm, in: Strategic Management Journal, Vol. 5, S. 171-180.

Williamson, Oliver E. (1967), Hierarchical control and optimum firm size, in: Journal of Political Economy, Vol. 75, S. 123-138.

Williamson, Oliver E. (1971), The vertical integration of production: market failure considerations, in: American Economic Review, Vol. 61, S. 112-123.

Williamson, Oliver E. (1975), Markets and Hierarchies: Analysis and Antitrust Implications. New York.

Williamson, Oliver E. (1979), Transaction Costs Economics: The Governance of Contractual relations, in: Journal of Law and Economics, Vol. 22, S. 233-262.

Williamson, Oliver E. (1985), The Economic Institutions of Capitalism, New York. 


\section{Vertikale Integration und Unternehmenserfolg: eine empirische Untersuchung in der Formel 1}

\section{Zusammenfassung}

Die neue Theorie der Unternehmung besagt, dass unter bestimmten Bedingungen vertikal integrierte Unternehmen effizienter arbeiten als nicht integrierte Unternehmen. Dies ist unter anderem auf Unterinvestitionsprobleme bei Vorliegen hoher transaktionsspezifischer Investitionen und unzureichender Messbarkeit der Leistungen einzelner Akteure zurückzuführen. Im Rahmen der vorliegenden Untersuchung kann für den Formel 1-Rennsport gezeigt werden, dass vertikal integrierte Unternehmen Unterinvestitionsprobleme besser lösen und die Integrationsentscheidung den Unternehmenserfolg positiv beeinflusst.

\section{Summary}

According to the (new) theory of the firm vertically integrated firms might under certain conditions outperform disintegrated firms. In particular, in a situation with high transaction specific investments and measurement problems concerning individual performance an underinvestment can arise. In this paper we show for Formula One motor racing that integrated firms solve arising underinvestment problems more efficiently than disintegrated firms and that the decision to integrated positively affect the firm’s performance. 